\title{
Single-point mutations in $Q \beta$ virus-like particles change binding to cells
}

Marisa L. Martino, ${ }^{1 \dagger}$ Stephen N. Crooke, ${ }^{2 \S}$ Marianne Manchester, ${ }^{3 \uparrow}$ M.G. Finn* ${ }^{1,2}$

${ }^{1}$ Department of Chemistry, The Scripps Research Institute, La Jolla, California 92037, USA

${ }^{2}$ School of Chemistry and Biochemistry, School of Biological Sciences, Georgia Institute of

Technology, Atlanta, Georgia 30332, USA

${ }^{3}$ Skaggs School of Pharmacy and Pharmaceutical Sciences, University of California, San Diego, CA 92093, USA. Present address: Roche Pharmaceuticals, Grenzacherstasse 124, 4070 Basel Switzerland

$\dagger$ Present address: Thermo Fisher Scientific, 5823 Newton Dr., Carlsbad, CA 92008, USA

$\S$ Present address: Viral Vaccine Preventable Diseases Branch, National Center for Immunization and Respiratory Diseases, Centers for Disease Control and Prevention, Atlanta, GA, USA

" Present address: Roche Pharmaceuticals, Grenzacherstasse 124, 4070 Basel, Switzerland

\section{Supporting Information}

\section{Primers}

Table S1. Complete list of primers used in molecular cloning experiments. Bold underlined regions are restriction sites with their respective overhangs italicized. Site-directed mutagenesis regions are indicated in blue italics with specific point mutations indicated in lowercase italics.

\begin{tabular}{|c|c|}
\hline Primer Name & Primer Sequence 5' - 3' \\
\hline CP-NcoI-F & CATGCCATGGCAAAATTAGAGACTGGTACTTTAGGTAACATC \\
\hline CP-XhoI-R & $\begin{array}{l}\boldsymbol{C T G C T C G A G T T A A T A C G C T G G G T T C A G C T G A T C ~} \\
\end{array}$ \\
\hline K2E-F & CATGCCATGGCA $g A A T T A G A G A C T G T T A C T T T A G G T A A C A T C$ \\
\hline K2Q-F & CATGCCATGGCA $c A g T T A G A G A C T G T T A C T T T A G G T A A C A T C$ \\
\hline K13E-F & GTAACATCGGGgAAGATGGA \\
\hline K13E-R & TCCATCTTCCCCGATGTTAC \\
\hline K13Q-F & GTAACATCGGGcAgGATGGA \\
\hline K13Q-R & TCCATC $c T g C C C G A T G T T A C$ \\
\hline K16E-F & GATGGA $g A A$ CAAACTCTGGTCC \\
\hline K16E-R & GGACCAGAGTTTGTTCTCCATC \\
\hline K16Q-F & GATGGAcAgCAAACTCTGGTCC \\
\hline K16Q-R & GGACCAGAGTTTGcTgTCCATC \\
\hline K16F-F & GAAAGATGGA $t t t C A A A C T C T G G T$ \\
\hline K16F-R & ACCAGAGTTTGaaaTCCATCTTTC \\
\hline K16Y-F & GAAAGATGGAtatCAAACTCTGGT \\
\hline K16Y-R & GAAAGATGGA $a t a$ CAAACTCTGGT \\
\hline K46E-F & GCGCTGGAG $g A A C G T G T T A C C G T$ \\
\hline K46E-R & GCGCTGGAG $g A A C G T G T T A C C G T$ \\
\hline K46Q-F & GCTGGAGcAgCGTGTTACCG \\
\hline K46Q-R & GGTAACACGcTgCTCCAGCG \\
\hline
\end{tabular}




\section{Chemical modification of VLPs}

Figure S1. (a) LC-ToF analysis of wild-type $\mathrm{Qb}$ functionalized with azide linker 2. From left to right, the labeled peaks represent $0,1,2$, 3 , and 4 acylated lysines per coat protein. (b) MALDI-MS analysis of T93@A48880.

(c) LC-ToF analysis of T93(N3)360@(A488)80.
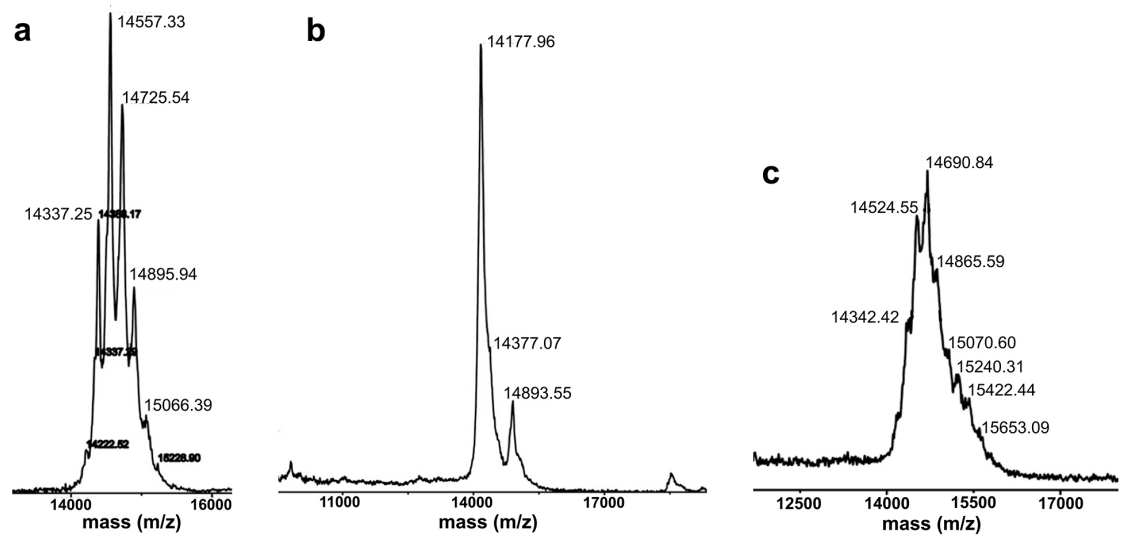

Figure S2 . (left) Sizeexclusion chromatography (Superose 6) of T93@488 particles after purification. (right) UV-vis absorption spectrum of the resulting particles at $0.9 \mathrm{mg} / \mathrm{mL}$ concentration.
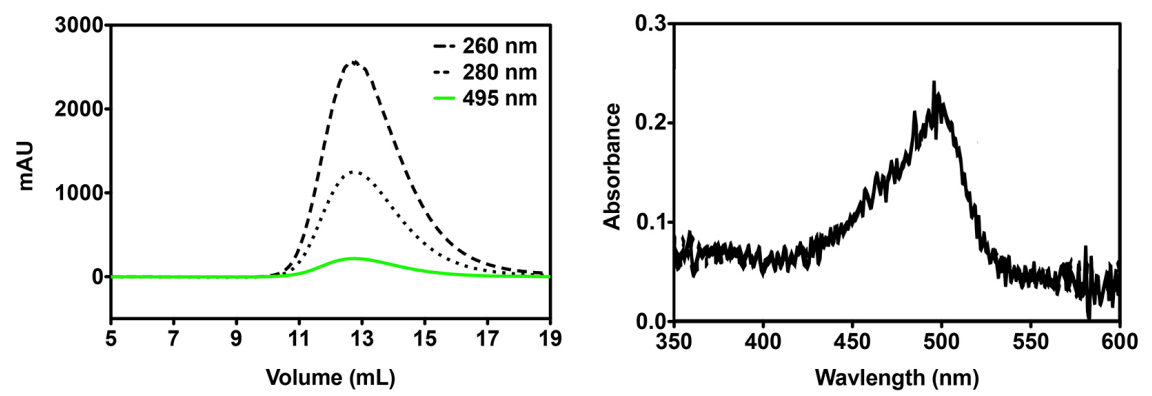

Figure S3. (a) Uv-vis absorbance spectra of the indicated particles labeled with the indicated dye. The numerical values associated with each curve are the average number of dye molecules attached per particle, deriving from reactions at different concentrations of NHS ester reagent. The samples used for the studies described displayed 20-25 dyes per particle. (b) Size-exclusion chromatography (Superose 6) of K46Q IR800cw particles, as a representative sample. (c) Dynamic light scattering of K46Q IR800 cw particles as a representative sample $(0.1 \mathrm{mg} / \mathrm{mL})$.
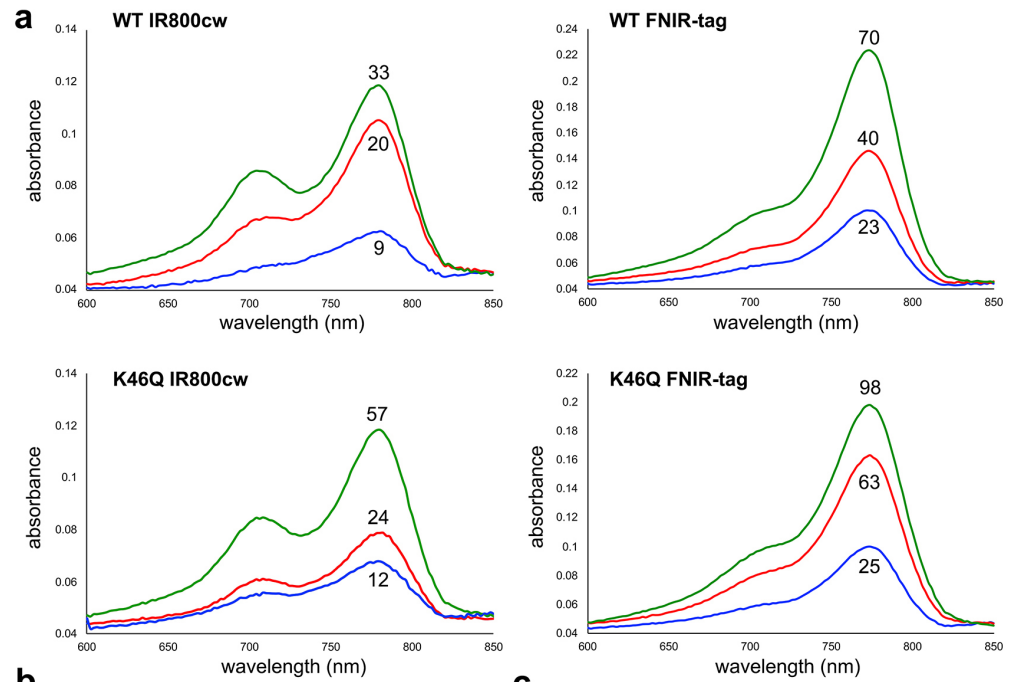

b
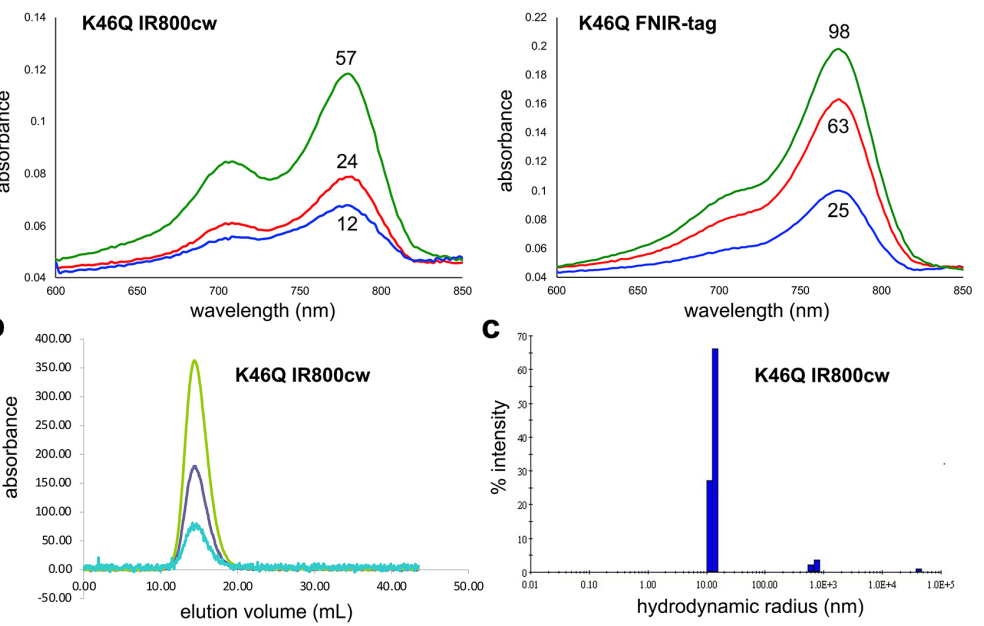


\section{TEM Images}
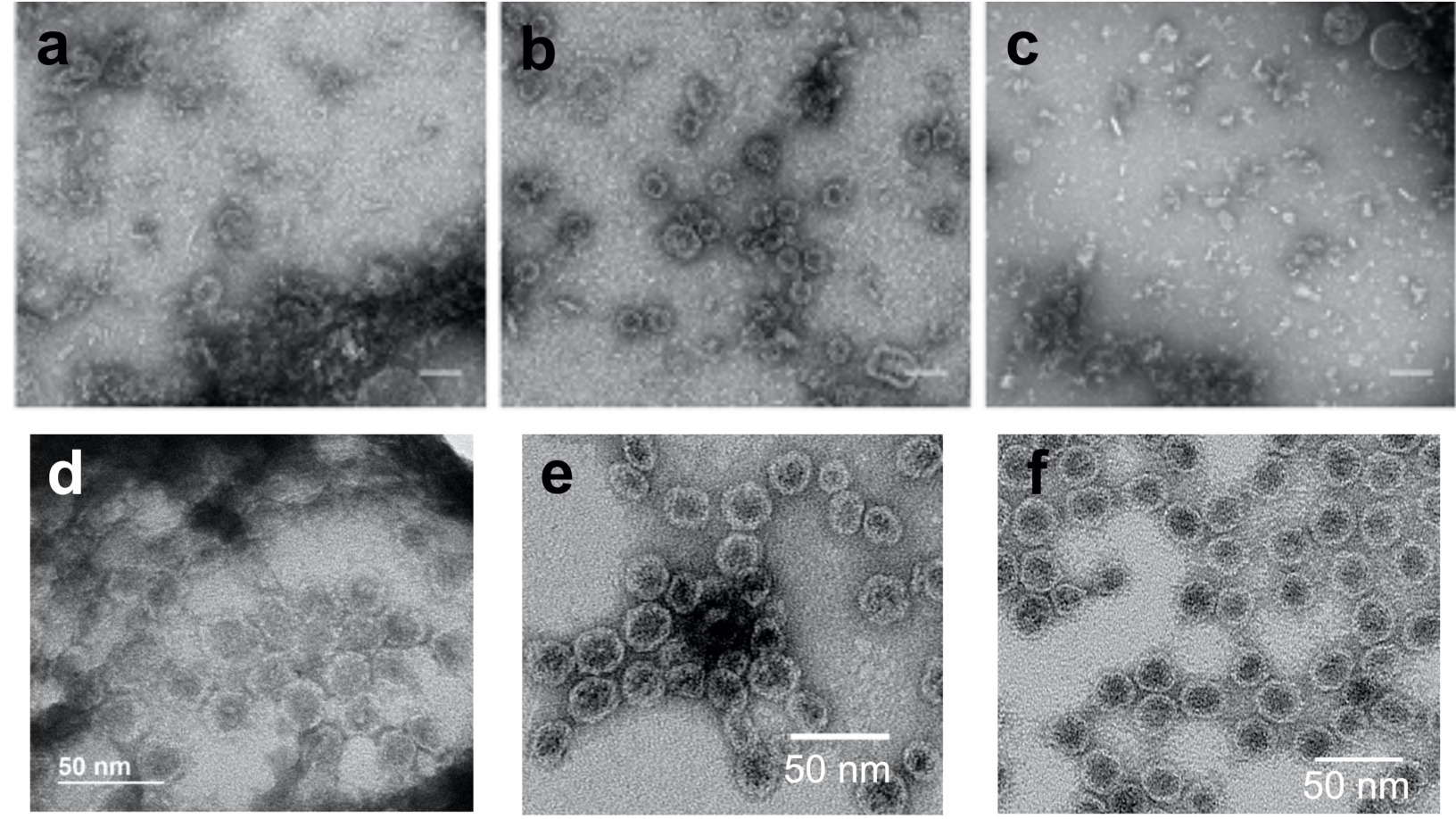

Figure S4. Negative stained transmission electron microscopy of: (a-c) cleared bacterial lysates from expression of (a) K2E, (b) K46E, and (c) K13E/K16E VLPs; purified (d) K2Q, (e) K16E, (f) K46Q

\section{ELISA Analysis}

Figure S5. Binding of anti$\mathrm{Q} \beta$ antibody to different VLP constructs. $\operatorname{LogEC} 50$ values were calculated using sigmoidal doseresponse non-linear regression analysis with GraphPad Prism software (GraphPad Software, San Diego, CA).

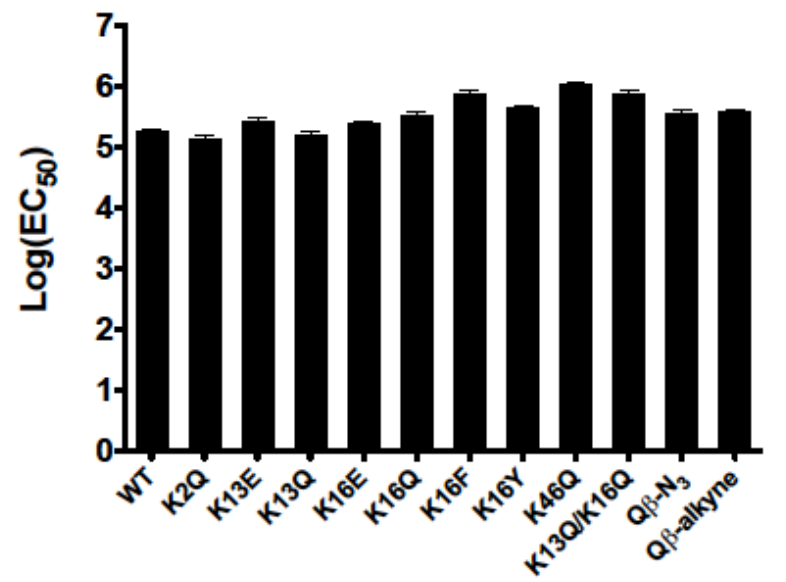

\begin{tabular}{|c|c|c|}
\hline & \multicolumn{2}{|c|}{$\log \left(E C_{50}\right)$} \\
\hline & Mean & SEM \\
\hline WT & 5.29 & 0.01 \\
\hline K2Q & 5.17 & 0.02 \\
\hline K13E & 5.45 & 0.02 \\
\hline K13Q & 5.24 & 0.01 \\
\hline K16E & 5.41 & 0.02 \\
\hline K16Q & 5.55 & 0.02 \\
\hline K16F & 5.91 & 0.02 \\
\hline K16Y & 5.67 & 0.02 \\
\hline K46Q & 6.06 & 0.01 \\
\hline K13Q/K16Q & 5.91 & 0.01 \\
\hline $\mathrm{Q} \beta-\mathrm{N}_{3}$ & 5.59 & 0.02 \\
\hline Qß-alkyne & 5.62 & 0.01 \\
\hline
\end{tabular}

VLP construct 
Figure S6. Optimization of blocking buffers used in VOPBA analysis of Q $\beta W T$ particles binding to A431 PM fractions. From left to right, blocking buffers are $5 \%$ milk, undiluted SuperBlock ${ }^{\mathrm{TM}}$ (PBS), 3\% goat serum, and 3\% BSA. A major Q $\beta$ binding protein is detected at $35 \mathrm{kDa}$ when milk, goat serum, and BSA are used as blocking buffers.

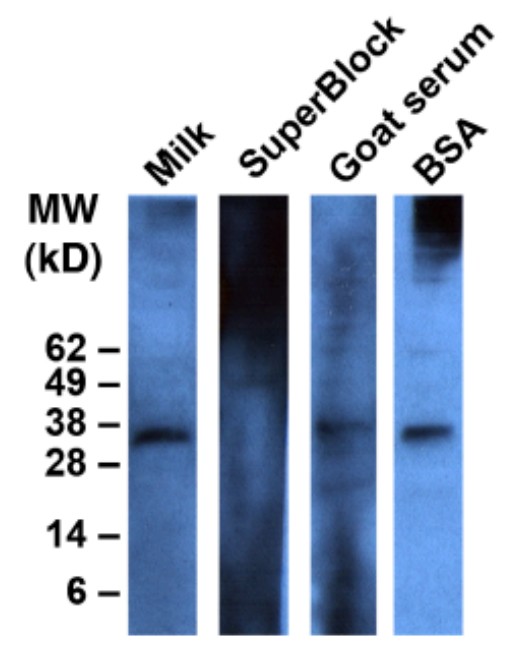

